\title{
Distinguishing between spatial coherence and temporal coherence of phonons
}

\author{
Benoit Latour \\ Division of Engineering and Applied Science, California Institute of Technology, Pasadena, California 91125, USA \\ and Laboratoire EM2C, CNRS, CentraleSupélec, Université Paris-Saclay, Grande Voie des Vignes, 92295 Châtenay-Malabry, France \\ Yann Chalopin* \\ Laboratoire EM2C, CNRS, CentraleSupélec, Université Paris-Saclay, Grande Voie des Vignes, 92295 Châtenay-Malabry, France
}

(Received 12 December 2016; published 30 June 2017)

\begin{abstract}
Coherent phonon transport is regarded as a promising strategy for controlling thermal properties in solids using the wave nature of phonons. However, no clear distinction between the spatial and temporal phonon coherence has been accounted for and a formalism that quantifies these two effects is still to be found. In this work, we propose a statistical approach for calculating the spatial and temporal coherence spectra using molecular dynamics simulations. We provide a microscopic assessment of these properties and we theoretically demonstrate that, while temporal and spatial coherence can be analytically related under specific conditions, they represent two characteristic lengths that set apart different physical effects. The former is associated with the phonon mean free path while the latter can be regarded as a measure of localization, representing the spatial extension of phonon wave packets. This provides a framework to engineer heat conduction in solids by quantitatively revealing the wave/particle nature of the vibrational modes.
\end{abstract}

DOI: 10.1103/PhysRevB.95.214310

\section{INTRODUCTION}

The concept of mean free path, which was first introduced by Clausius [1] in 1857, remains essential nowadays to formulate a microscopic description of heat conduction. In dielectric crystals, phonons, which correspond to collective excitations of atomic nuclei under the form of vibrational modes, are the main energy carriers. In bulk materials, the finite value of their mean free path is dictated by multiple-phonon processes arising from the nonlinear atomic interactions [2]. Such a particlelike description of heat conduction in solids, formulated in the light of the Boltzmann transport theory introduced by Peierls [3], has allowed to tailor the thermal properties of materials with dimensions below the bulk mean free path. This has opened a window into a wide range of applications, including energy harvesting [4,5] or thermal management $[6,7]$.

It is interesting to note that, although the particle aspect of phonons is well handled [8-12], there remains plenty of room for progress in engineering thermal properties of solids through the wave nature of heat conduction. Localization phenomena in disordered materials is a broad research topic [13-23] where interference phenomena are central. The proportion of vibrational modes affected by localization is still an ongoing work, which requires to take into consideration the wave nature of the atomic motion. In addition, recent advances in materials science have allowed to engineer the density of states and the group velocity of thermal phonons via interference phenomena in superlattices [24,25] (SLs). Both experimental [26-30] and numerical [31-43] works stated the importance of coherence on the thermal properties of SLs, as both a wave and a particle description of phonons are necessary to explain most of the results. So far, no clear theoretical framework has allowed to establish quantitatively to what extent phonon modes transit

\footnotetext{
*yann.chalopin@cnrs.fr
}

from a pure plane-wave behavior toward a diffusive pointlike particle.

To address this problem, we propose to revisit heat conduction in solids from the angle of spatial and temporal coherence of atomic vibrations. From the statistical approach based on equilibrium molecular dynamics simulations, we demonstrate that the study of coherence reveals characteristic length scales that allow us to form a complete description of the various transport regimes that occur and sometimes coexist in any crystal. In a first discussion, we demonstrate that a finite temporal and spatial coherence originate from the nonlinearity in the lattice and correspond respectively to the mean free path and to the distance by which interference effects appear. In addition, we show that the spatial coherence represents a measure of localization of the atomic motion, representing the spatial extension of phonon wave packets. Hence we introduce a complex wave vector and frequency to extend the description of the phonon dispersion relations to nonlinear crystals. We next investigate heat transport in one-dimensional SLs to present ballistic transport, interface scattering, and confinement from the point of view of the spatial and temporal coherence. We finally generalize these results to bulk materials (single-layer suspended graphene) and validate our approach by comparison with recent experimental measurements.

\section{SPATIAL AND TEMPORAL COHERENCE OF THERMAL PHONONS}

\section{A. Theoretical framework}

Coherence is related to the persistence of a physical process which can be captured by means of correlations [44]. By adopting a statistical approach, we have related the thermal fluctuations of the atomic velocities to the spatial and temporal correlation of heat waves in a $d$-dimensional crystal with $n_{u c}$ atoms in the unit cell. $\left\{\vec{a}_{i}\right\}_{i \in\{1, d\}}$ are the primitive vectors of 
the Bravais lattice. We note $\vec{R}_{k}^{0}=\sum_{i=1}^{d} c_{i}^{k} \vec{a}_{i}$, with $c_{i}^{k} \in \mathbb{Z}$, the position of the cell $i .\left\{\vec{b}_{i}\right\}_{i \in\{1, d\}}$ are the primitive vectors of the Brillouin zone. $\vec{v}\left(t_{i}, \overrightarrow{R_{j}^{0}}, b\right)$ corresponds to the velocity of the atom $b$ in the unit cell $j$ at time $i \Delta t$. By assuming a stationary field, a mutual coherence function $T\left(\tau, \overrightarrow{R_{k}^{0}}, \overrightarrow{R_{l}^{0}}\right)$ can be constructed. It corresponds to the time cross-correlation of the velocity field at $\vec{R}_{k}^{0}$ and $\overrightarrow{R_{l}^{0}}$ :

$$
T\left(\tau, \overrightarrow{R_{k}^{0}}, \vec{R}_{l}^{0}\right)=\frac{1}{2} \sum_{b=1}^{n_{u c}} m_{b}\left\langle\vec{v}\left(t, \vec{R}_{k}^{0}, b\right) \vec{v}\left(t+\tau, \vec{R}_{l}^{0}, b\right)\right\rangle_{t},
$$

where $\langle\ldots\rangle_{t}$ stands for the time correlation operator. The factor $m_{b} / 2$ is used to have the same form as a kinetic energy. The cross-spectral density function is defined as the time Fourier transform of the mutual coherence function:

$$
T\left(\omega, \overrightarrow{R_{k}^{0}}, \vec{R}_{l}^{0}\right)=\frac{1}{2} \sum_{b=1}^{n_{u c}} m_{b} \vec{v}^{*}\left(\omega, \overrightarrow{R_{k}^{0}}, b\right) \vec{v}\left(\omega, \vec{R}_{l}^{0}, b\right),
$$

where ${ }^{*}$ corresponds the complex conjugate. The system is observed at equilibrium, so the field is homogeneous. We introduce an additional operator, which in addition to including the temporal cross-correlation of the velocity also assumes the spatial one. The spatial correlation direction, noted $\vec{R}$, belongs to the Bravais lattice. Thus we have

$$
T(\omega, \vec{R})=\frac{1}{2} \sum_{b=1}^{n_{u c}} m_{b}\left\langle\vec{v}^{*}\left(\omega, \overrightarrow{R^{0}}, b\right) \vec{v}\left(\omega, \overrightarrow{R^{0}}+\vec{R}, b\right)\right\rangle_{\vec{R}^{0}},
$$

with $\langle\ldots\rangle_{\vec{R}^{0}}$, the spatial cross-correlation operator.

Finally, the spatial Fourier transform of $T(\omega, \vec{R})$ gives the spectral energy density, as proved in Ref. [51]:

$$
T(\omega, \vec{k})=\sum_{v} \frac{1}{2}|\dot{q}(\omega, \vec{k}, v)|^{2},
$$

where $\dot{q}(\omega, \vec{k}, \nu)$ corresponds to the time derivative of the normal coordinates of the mode $(\vec{k}, v)$ and $v$ denotes the branch in the dispersion relations.

\section{B. Spatial and temporal coherence in anharmonic materials}

Using the previously established theoretical framework, the effect of the anharmonicity on the coherence properties of a crystal is illustrated using molecular dynamics. For the sake of clarity, we consider a one-dimensional SL, composed of two diatomic chains, named $\mathrm{A}$ and $\mathrm{B}$, as depicted on Fig. 1. We use a Lennard-Jones potential $E(r)$ [45] to include anharmonic interactions in the system:

$$
E(r)=4 \epsilon\left[\left(\frac{\sigma}{r}\right)^{12}-\left(\frac{\sigma}{r}\right)^{6}\right],
$$

with $\epsilon=5 \epsilon_{A r}$ and $\sigma=\sigma_{A r}$. The same potential parameters are applied to all the atoms of the superlattice; only their mass differs. For material 1, $m_{1}=m_{A r}$ and $m_{2}=2 m_{A r}$, where $m_{A r}$ is the atomic mass of the $\operatorname{argon}$ atom. For material $2, m_{3}=$ $3 / 2 m_{A r}$ and $m_{4}=3 m_{A r}$. The shortest superlattice period $d_{\mathrm{SL}}$ corresponds to the stacking 1212 and is equal to $1.54 \mathrm{~nm}$. For illustration, the superlattice with $d_{\mathrm{SL}}=6.16 \mathrm{~nm}$ has a stacking 11112222. The molecular dynamics time step is set to $3 \mathrm{fs}$. The
$1212 \ldots 1212$
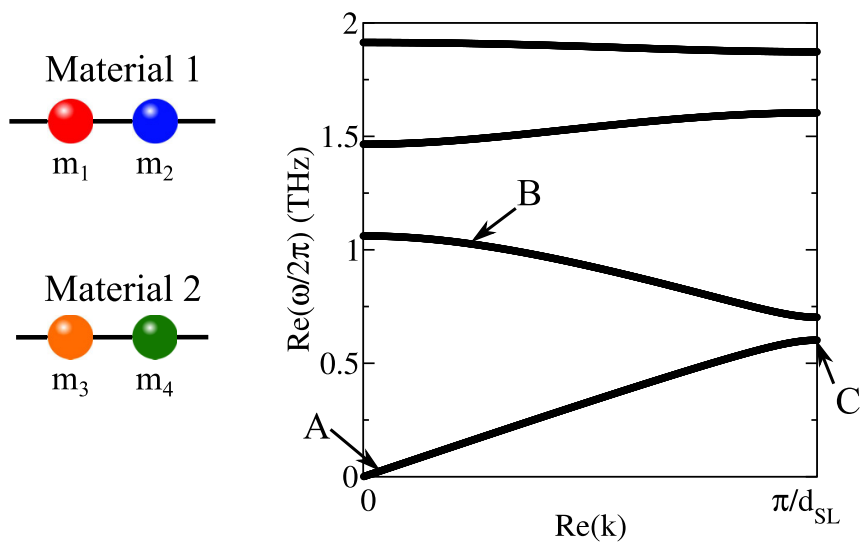

FIG. 1. Presentation of the one-dimensional superlattices composed of materials 1 and 2 and its dispersion relations computed from the spectral energy density when the period thickness $d_{\mathrm{SL}}$ is equal to $1.54 \mathrm{~nm}$. The letters A, B, and C on the dispersion relations label the simulated wave packets.

structure is first relaxed in the NPT ensemble for $10^{7}$ steps at 0 bar. The equilibrium distance between atoms, noted $a$, is equal to $0.382 \mathrm{~nm}$ at $10 \mathrm{~K}$. Then, the simulations are done in the NVE ensemble and the velocities are saved every 64 steps during 2097152 steps. An average on 20 independent simulations was done to compute the spectral energy density and the cross-spectral density function. Figure 1 presents the dispersion relations of this one-dimensional superlattices with $d_{\mathrm{SL}}=1.54 \mathrm{~nm}$, computed from the spectral energy density.

We present in Fig. 2(b) the impact of the interatomic potential anharmonicity, as shown in Fig. 2(a), on the spectral energy density $T(\omega, \vec{k})$ of this one-dimensional superlattice. For clarity reasons, only the low-frequency part of the acoustic branch is represented. In the harmonic approximation, the phonon dispersion relations have a discrete structure composed of Dirac functions, which tends to a widthless continuous curve for an infinite system. The nonlinear interactions due to the Lennard-Jones potential lead to a broadening of both the frequency $\omega$ and wave vector $k$ energy distribution. We now focus on a single mode $\left(k_{0}, \omega_{0}\right)$ of this dispersion relation. When looking at the cross-spectral density function $T\left(\omega_{0}, \vec{R}\right)$ in Fig. 2(c), we conclude that the corresponding wave of this mode is delocalized in the harmonic approximation whereas in the anharmonic case, $T\left(\omega_{0}, \vec{R}\right)$ decays exponentially as the two point correlation distance increases. This decay defines the spatial coherence length $l_{c}\left(\omega_{0}\right)$.

When the Lennard-Jones potential is truncated at the second-order [harmonic approximation as shown in Fig. 2(a)], the spectral energy density $T\left(\omega_{0}, k\right)$ should exhibit a Dirac profile along the wave-vector axis, as it is depicted in Fig. 2(d). This Dirac profile corresponds to the spatial Fourier transform of the plane wave. When the anharmonic potential is considered, $T\left(\omega_{0}, k\right)$ possesses a Lorentzian profile, with the full width at half maximum corresponding to the spatial coherence length. There is a significant advantage of choosing the cross-spectral density function over the spectral energy density: one does not require the explicit knowledge of 
(a)

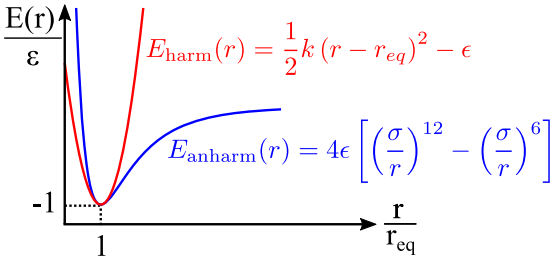

Harmonic potential

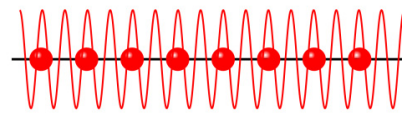

Anharmonic potential

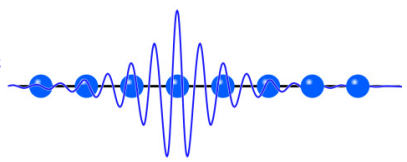

(b)

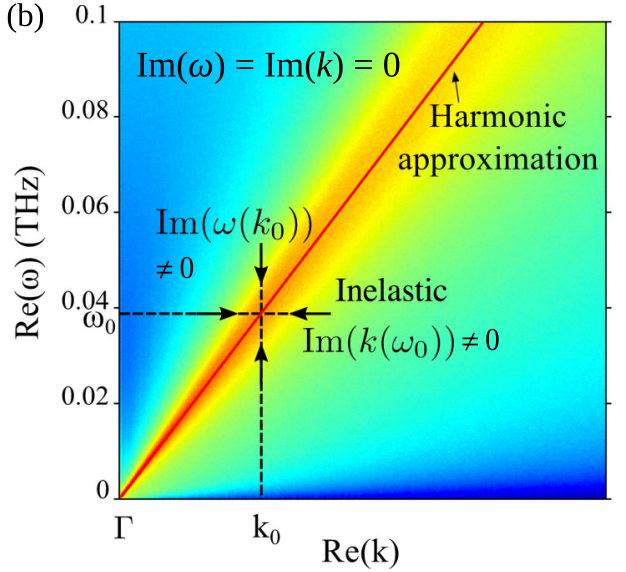

(c)

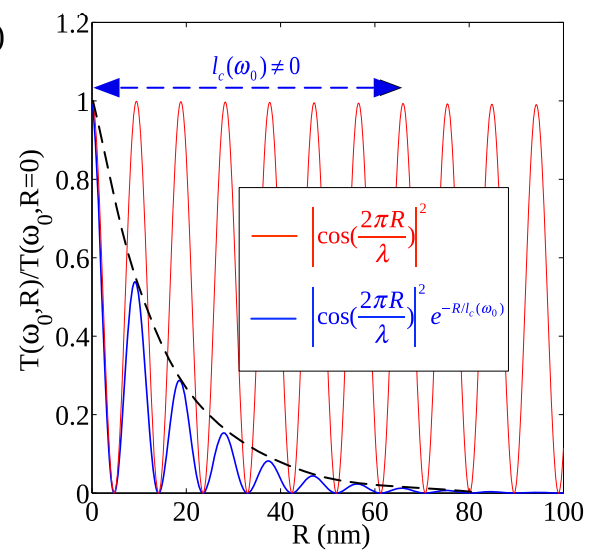

(d)

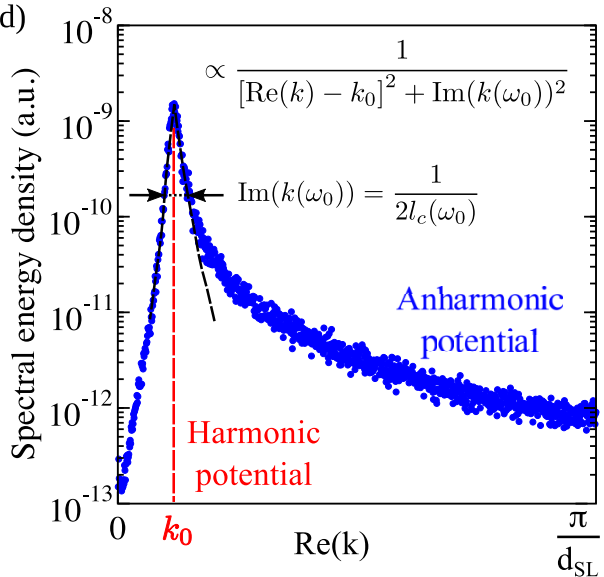

FIG. 2. Spatial coherence of thermal phonons in anharmonic materials. (a) Anharmonic Lennard-Jones potential (blue) vs harmonic approximation (red). (b) Spectral energy density $T(\omega, k)$ of the one-dimensional SL chain with $d_{\mathrm{SL}}=1.54 \mathrm{~nm}$ at $T=10 \mathrm{~K}$ for low-frequency modes of the acoustic branch. In the harmonic crystal, $T(\omega, k)$ reduces to a line (red), whereas in anharmonic systems, a frequency and wave vector broadening appear. The color map represents the intensity of the spectral energy density, from low intensity (dark blue) to high intensity (dark red). (c) Cross-spectral density function $T\left(\omega_{0}, R\right)$ computed from molecular dynamics for the mode $\left(\omega_{0}, k_{0}\right)$ with an exponential decay dictated by the spatial coherence length $l_{c}\left(\omega_{0}\right)$ in the anharmonic system (blue) and the harmonic one (red). (d), Wave vector dependence of the spectral energy density at $\omega=\omega_{0}$ showing a width related to the imaginary part of the wave vector.

the eigenvectors to measure the spatial coherence length, which explains the difference of the spatial coherence spectra obtained with $T(\omega, R)$ and $T(\omega, k)$ later in the manuscript.

This first statement leads to an important conclusion: in realistic nonlinear lattices, phonons are not simple delocalized plane waves with an infinite spatial coherence length but rather wave packets with a finite spatial extension, as it has been recently experimentally demonstrated [46]. In a similar fashion, the temporal coherence can be estimated from the frequency broadening of the spectral energy density. It allows us to extract the phonon relaxation time, which corresponds to the inverse of the mean free path $\Lambda\left(\omega_{0}\right)$ divided by the phonon group velocity. Again, due to the anharmonic nature of the lattice, phonons have a finite relaxation time. In this paper, we argue that these finite lengths $\left(\Lambda, l_{c}\right)$ reflect the particle and wave nature of phonons when compared concomitantly to the system characteristic length.

A complete theory of phonon coherence has never been introduced so far. To properly capture those novel length scales in nonlinear lattices, we propose to introduce an imaginary part to the frequency [47-49] $\omega=\omega_{0}+i \operatorname{Im}(\omega)$ and the wave vector $k=k_{0}+i \operatorname{Im}(k)$ for each mode. The spatial coherence length is defined as $l_{c}\left(\omega_{0}\right)=1 /\left[2 \operatorname{Im}\left(k\left(\omega_{0}\right)\right)\right]$ and the mean free path as $\Lambda\left(k_{0}\right)=v_{g}\left(\omega_{0}, k_{0}\right) /\left[2 \operatorname{Im}\left(\omega\left(k_{0}\right)\right)\right]$, where $v_{g}\left(\omega_{0}, k_{0}\right)$ corresponds to the group velocity of the mode $\left(\omega_{0}, k_{0}\right)$. This generalization encompasses the harmonic approximation, where only the real parts $\omega_{0}$ and $k_{0}$ are nonzero. Previous works $[31,50]$ have addressed the importance of the spatial coherence but both of these pioneer works present only part of the elements to form a complete theory of phonon coherence because of a confusion between the temporal and spatial coherence.

\section{COMPARISON BETWEEN THE MEAN FREE PATH AND THE SPATIAL COHERENCE LENGTH}

\section{A. One-dimensional superlattices}

To differentiate the physics captured by the spatial and temporal coherence, we have compared the phonon mean free path $\Lambda$ and the spatial coherence length $l_{c}$ for the 1D SLs previously presented for different period thickness. The number of atoms is kept constant at 8000 when changing the superlattice period in order to keep the same number of modes in the system. For a period of $d_{\mathrm{SL}}=1.54 \mathrm{~nm}$, Fig. 3(a) shows 

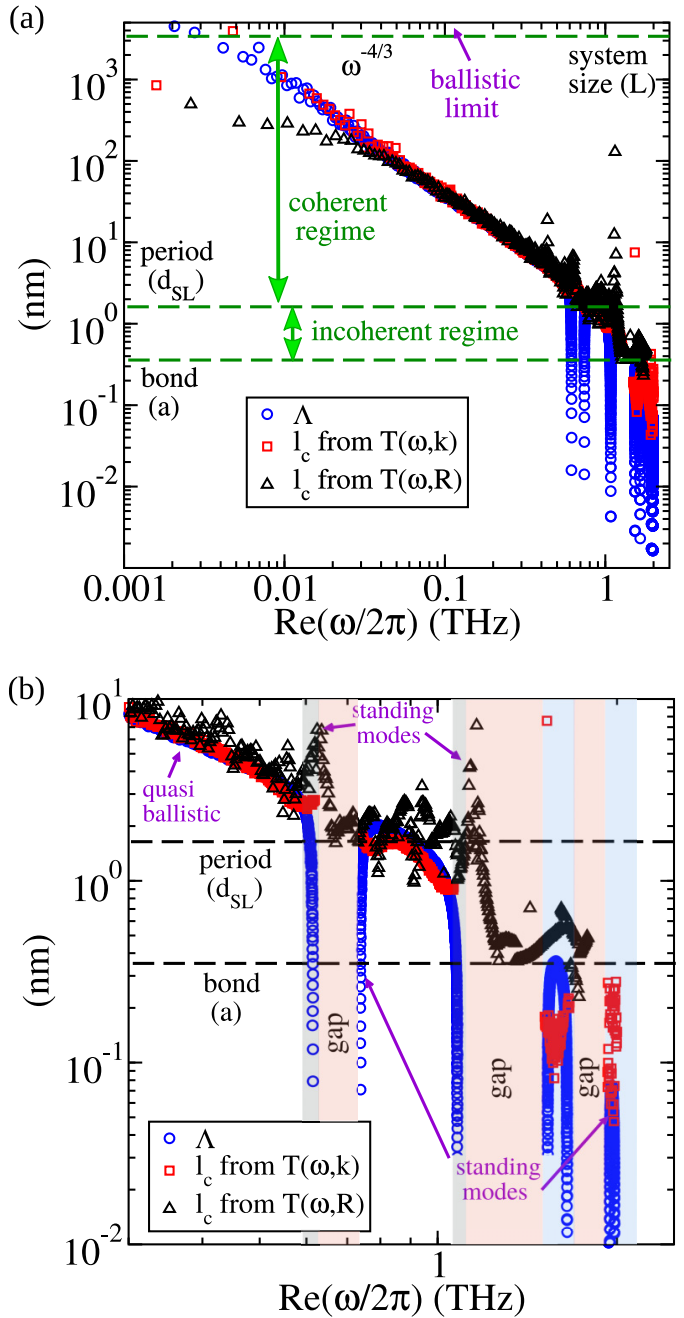

FIG. 3. Temporal (blue) and spatial (red and black) coherence in the SL with $d_{\mathrm{SL}}=1.54 \mathrm{~nm}$. (a) Comparison of the phonon mean free path $\Lambda$ and the spatial coherence length $l_{c}$ computed from the spectral energy density $T(\omega, k)$ and from the cross-spectral energy density $T(\omega, R)$. The spatial coherence length and the mean free path describe the coherent and incoherent regimes. The discrepancy between $T(\omega, R)$ and $T(\omega, k)$ indicates that the assumption of a SL Brillouin zone is no longer valid as nonlinear modes are observed in the frequency gaps. (b) The spatial coherence length and the mean free path also describe additional transport regimes, such as quasiballistic transport or standing waves.

that the spatial coherence length of the low-frequency phonons matches their mean free path: the spatial extension of these modes thus corresponds to the distance between two successive scattering events. It is demonstrated analytically (as Ref. [51]) how $l_{c}$ and $\Lambda$ are associated. Provided that $\operatorname{Im}(\omega) \ll \operatorname{Re}(\omega)$ and $\operatorname{Im}(k) \ll \operatorname{Re}(k)$, the relation $v_{g}=\operatorname{Im}(\omega) / \operatorname{Im}(k)$ can be obtained, corresponding to $\Lambda(\omega)=l_{c}(\omega)$. The distance $l_{c}=\Lambda$ is much larger than the $\mathrm{SL}$ period $d_{\mathrm{SL}}$, indicating that the transport is ballistic (i.e., insensitive to reflection/transmission at the interfaces). Interestingly, at the edge and center of the first Brillouin zone $(0.6$ and $1.0 \mathrm{THz})$, we have observed two giant spatial coherence lengths much larger than the superlattice period. The corresponding $\Lambda$ falls to zero. This is consistent with the expected behavior of a standing wave [as highlighted in Fig. 3(b)] characterized by a nonzero spatial extension associated with a nonpropagative behavior. It reveals that spatial coherence can persist over distances as large as the system size (at $1 \mathrm{THz}$ ) in absence of any energy transport. Consequently, temporal (respectively, spatial) coherence supplies a complementary information qualifying the particle (respectively, wave) behavior of a phonon. Coherence should be sometimes disconnected from the concept of ballisticity. In the same line, Fig. 3(b) reveals the presence of phonon modes lying in the band gaps, with coherence lengths smaller than the SL period thickness. Here, the assumption of a SL phonon dispersion no longer holds. Considering that these modes are in a diffuse regime, their mean free path corresponds to one of the two materials in contact. In this incoherent regime, $\Lambda$ and $l_{c}$ have to be calculated for each material. In our system, both materials exhibit very close $l_{c}$ and an averaged value has been considered for the sake of the figure clarity. It should be mentioned as well that the acoustic mismatch also leads to evanescent waves. They form another class of nonpropagative modes spotted by a short (subperiod) spatial coherence length.

We have observed that the spatial coherence length does not depend on $d_{\mathrm{SL}}$ over the whole spectrum. However, dividing $l_{c}$ by $d_{\mathrm{SL}}$ [Fig. 4(a)] points up a frequency domain in which the spatial coherence length is smaller than the period thickness $\left(l_{c} / d_{\mathrm{SL}}<1\right)$. When increasing $d_{\mathrm{SL}}$, this frequency domain increases as well. Consequently, the distance between two interfaces forming the SL becomes progressively larger than the spatial extension of the phonons modes: reflection and transmission at the interfaces eventually occur. The calculation of the participation ratio (in the inset) shows that a smaller fraction of atoms in the unit cell of the superlattice is participating in the vibration modes when increasing $d_{\mathrm{SL}}$. This result is more pronounced at high frequencies, which is consistent with a broader spectrum of incoherent modes when increasing $d_{\mathrm{SL}}$. This corresponds to the transition from a coherent to an incoherent transport.

To visualize the different phonon transport regimes in these 1D superlattices, we have visualized the temporal and spatial structures of coherent and incoherent wave packets using wave packet simulations $[52,53]$. We chose the superlattice with period thickness $d_{\mathrm{SL}}=1.54 \mathrm{~nm}$. To generate these wave packets, we start from the equilibrium structure of the chain and initially displace each atom along the $x$ direction as

$$
u_{l, b}^{x}(t=0)=\frac{1}{\sqrt{M_{b}}} Q_{k_{0}, v} \epsilon_{k 0, v, b}^{x} e^{i k_{0}\left(x_{l}-x_{0}\right)} e^{-\frac{\left(x_{l}-x_{0}\right)^{2}}{l_{c}\left(\omega\left(k_{0}, v\right)\right)^{2}}},
$$

where $(l, b)$ refers to the $b$ th atom in the $l$ th unit cell (at the position $x_{l}$ ) of the chain with a mass $M_{b}$ and $Q_{k_{0}, v}$ to the amplitude of the wave. The spatial extension of the wave packet is usually a free parameter in this type of simulation. The eigenvector $\epsilon_{k 0, v, b}^{x}$ is computed from lattice dynamics. We argue here that the spatial extension is dictated by the spatial coherence length of the mode $l_{c}\left(\omega\left(k_{0}, v\right)\right)$. Here, we set $Q_{k_{0}, v}=10^{-5} \AA$. The initial velocities are computed following the standard procedure described elsewhere [53]. We have chosen three modes in the dispersion relations, labeled A, B, and C in Fig. 1. The properties of each wave packet are given in Table I. Movies of these wave packet simulations are provided in Ref. [51]. The wave packet A 

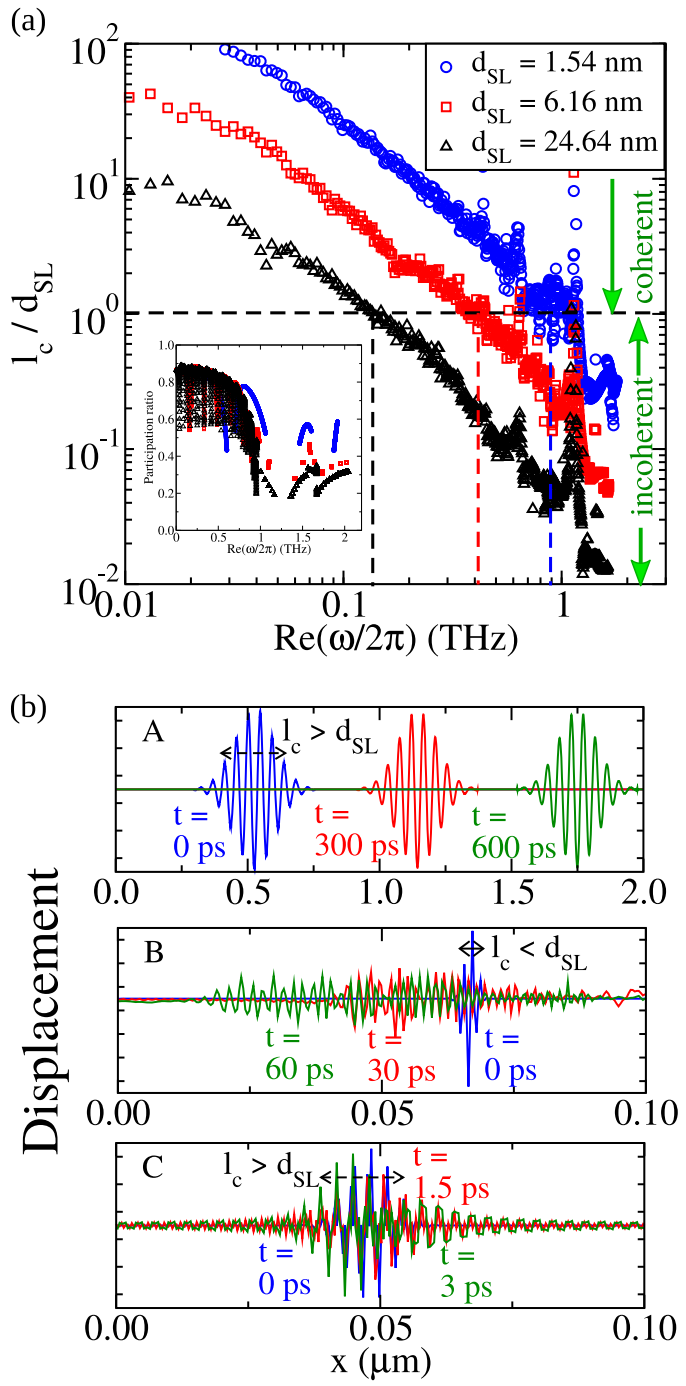

FIG. 4. (a) Dependence of the spatial coherence length $l_{c}$ normalized by the period thickness $d_{\mathrm{SL}}$ with $d_{\mathrm{SL}}$. When $l_{c} \geqslant d_{\mathrm{SL}}$, the transport of the modes is coherent. The dashed lines indicate the frequency cutoff above which the transport becomes incoherent for each SL. The inset provides the participation ratio for each $d_{\mathrm{SL}}$. (b) Wave packet dynamics simulations (mode A, B, and C in Fig. 1), respectively, illustrate the ballistic coherent transport, the interface scattering and the coherent standing mode.

represents a low-frequency phonon, which has been previously identified as ballistic in Fig. 3(a). The wave packet dynamics simulation [Fig. 4(b)] reveals a structure spreading over tens of periods that remains stable over time and confirms a ballistic transport. On the contrary, the optical mode (wave

TABLE I. Properties of the wave packets simulated in the 1D superlattices.

\begin{tabular}{lccc}
\hline \hline Wave packet & $k_{0}\left(2 \pi / d_{\mathrm{SL}}\right)$ & $\omega / 2 \pi(\mathrm{THz})$ & $l_{c}(\mathrm{~nm})$ \\
\hline $\mathrm{A}$ & 0.034 & 0.046 & 105 \\
$\mathrm{~B}$ & 0.1 & 1.04 & 1.8 \\
$\mathrm{C}$ & 0.5 & 0.60 & 6 \\
\hline \hline
\end{tabular}

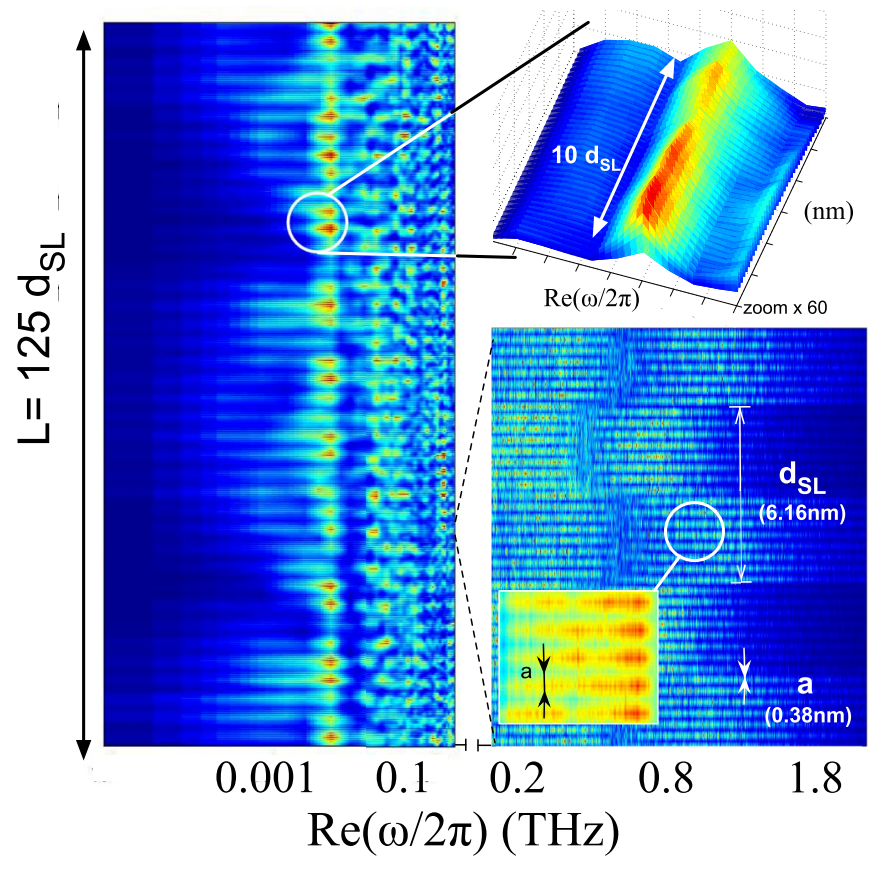

FIG. 5. Local density of states of phonons for the SL with $d_{\mathrm{SL}}=$ $6.16 \mathrm{~nm}$. In the coherent regime (below $0.12 \mathrm{THz}$ on the left), phonons are delocalized over several tens of periods. A zoom at the top-right part of the figure depicts this spreading. In the incoherent regime (above $0.3 \mathrm{THz}$ on the right), energy confinement can be observed in each layer of the SL as well as at the scale of a single atom (inset).

packet B) exhibits a coherence length close to $d_{\mathrm{SL}}$. With an initial negative group velocity direction, the wave packet undergoes multiple reflections and transmissions at interfaces, leading to a complex interference pattern with thermal energy propagating in both directions. We have finally turned to the case of the acoustic mode at the zone edge (wave packet C), characterized as a standing wave with a coherence length much larger than $d_{\mathrm{SL}}$. The simulation clearly reveals such a behavior, originating from the sum of two counter-propagative modes.

To end this analysis, we would like to confront the spatial coherence spectra to the local density of states (LDOS) of phonons to visualize how coherence is related to the spatial distribution of thermal energy. The diagonal elements of the cross-spectral density function already contains the information about the LDOS. Figure 5 distinguishes the phonon LDOS in a SL of $d_{\mathrm{SL}}=6.16 \mathrm{~nm}$ for the coherent and incoherent frequency bands. The low frequency phonons (below $0.12 \mathrm{THz}$ ) have been identified as ballistic and coherent and we observe a periodic localization of the thermal energy spreading over tens of SL unit cells (see the magnification on the right-hand side of the figure): this clearly indicates a wave behavior of these low frequency modes, which is consistent with the large wavelengths involved $\left(\sim 30 d_{\mathrm{SL}}\right)$. These modes are insensitive to the interfaces as $\lambda \gg d_{\mathrm{SL}}$ but also because $l_{c} \gg d_{\mathrm{SL}}$. Regarding the incoherent domain (above $0.3 \mathrm{THz}$ ), the LDOS allows to visualize two different length scales of energy confinement. The first one corresponds to a half period: each of the two materials forming the SL have different LDOS and part of the energy remains trapped in each layer leading to a Kapitza description of the interface resistance. At a smaller 


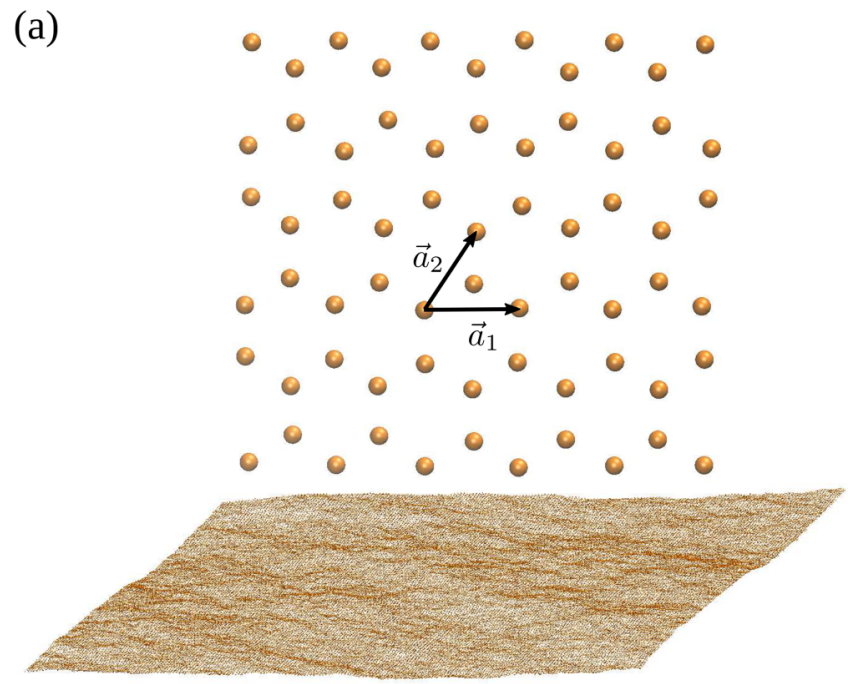

(b)

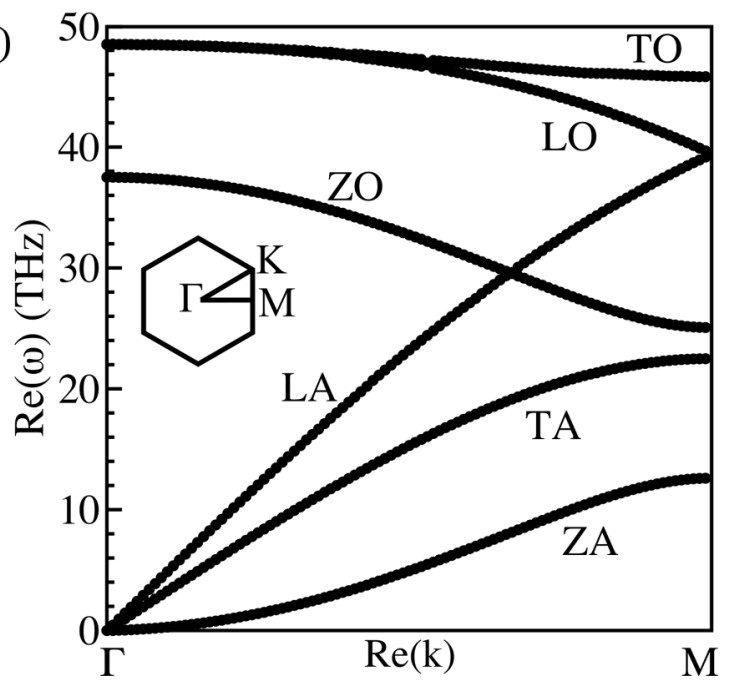

FIG. 6. Presentation of the single-layer graphene. (a) Atomistic structure of the single-layer graphene with the primitive vectors $\vec{a}_{1}$ and $\vec{a}_{2}$ and a snapshot of the sheet during the molecular dynamics simulation. (b) Dispersion relations in the $\Gamma \mathrm{M}$ direction for the singlelayer graphene from the spectral energy density.

scale (inset in the incoherent regime), the LDOS indicates another scale of energy confinement corresponding to the interatomic distance $a$ at the highest frequencies. This analysis of the LDOS is fully consistent with Fig. 3(b) for the modes with a localization length lying between $a$ and $d_{\mathrm{SL}}$.

\section{B. In single-layer graphene}

To further generalize these results, the coherence of thermal phonons has been investigated for a suspended graphene monolayer and compared to recent experimental measurements of the spatial coherence length [54]. Single layer graphene was simulated using the optimized Tersoff potential [55]. Figure 6(a) shows the hexagonal structure of the graphene monolayer. The size of the simulated layer is 200 unit cells along each of the primitive vector $\vec{a}_{1}$ and $\vec{a}_{2}$. As large spatial coherence lengths are expected in graphene, the temperature is set to $1500 \mathrm{~K}$ to obtain a dominant coherence length tractable (a)

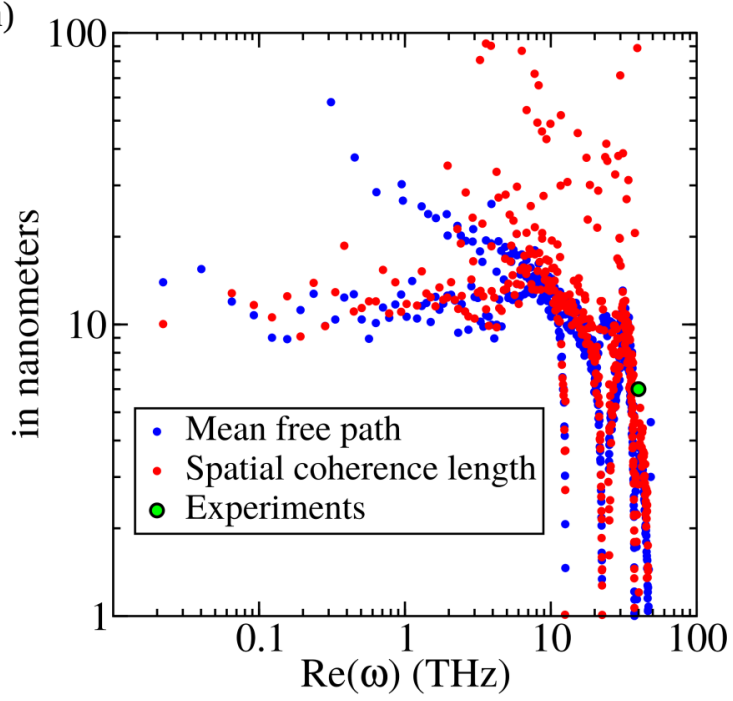

(b)

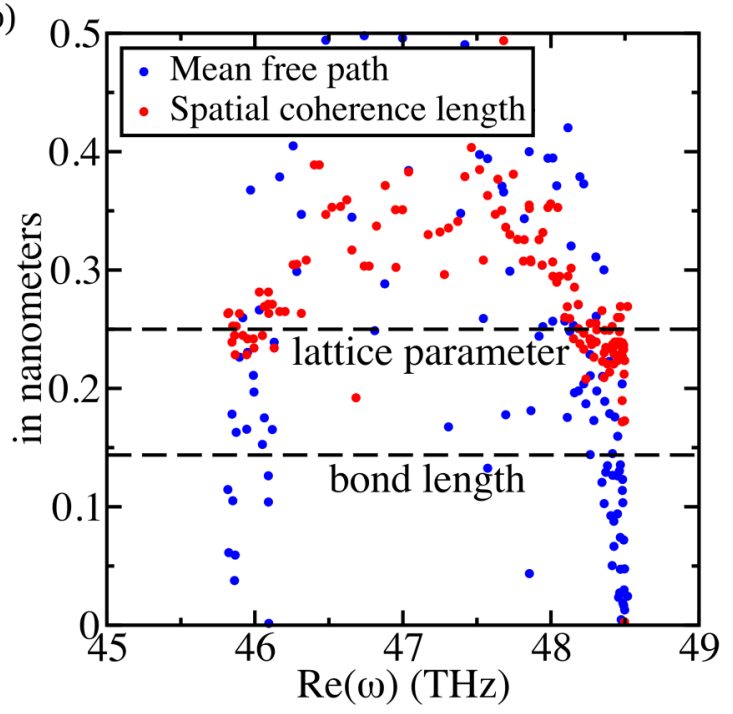

FIG. 7. Temporal and spatial coherence in a single-layer graphene at $1500 \mathrm{~K}$. (a) Comparison between the mean free path and the spatial coherence length for all the modes in the $\Gamma \mathrm{M}$ direction. The experimental measurement has been done by Katayama et al. [54]. (b) Comparison between the mean free path and the spatial coherence length for the transverse optical (TO) branch in the $\Gamma \mathrm{M}$ direction.

considering the system size. The molecular dynamics time step is 0.5 fs. The velocity field is registered every 16 steps, during 131072 steps. Twenty independent simulations were carried to get statistically satisfying results. We note that data for the spatial coherence length are more scattered than for the mean free path due to the limited wave vector resolution (100 points for the wave vector resolution against 4097 points for the frequency resolution). Figure 6(b) presents the phonon dispersion relations along the $\Gamma \mathrm{M}$ direction computed from the spectral energy density.

Figures 7(a) and 7(b) compare the mean free path and the spatial coherence length in the $\Gamma \mathrm{M}$ direction of the Brillouin zone. As observed for the case of SLs, the similarity between the mean free path and the spatial coherence length spectra is remarkable. Most of the high group velocity modes have a spatial extension comparable to the distance over which 
they propagate without scattering. As it is highlighted in Fig. 7(b), the transverse optical (TO) branch exhibits a mean free path which tends to zero at the center and the edge of the Brillouin zone. The spatial coherence length converges to a value close to the lattice parameter $(2.492 \AA)$, which plays the same role than the SL period in the previous systems. Again, spatial and temporal coherence deviate from each other when the group velocity of the branch tends to zero. Katayama et al. [54] have reported a spatial extension of thermal optical phonons near the $K$ point in the Brillouin zone of about 6 $\mathrm{nm}$ for a single graphene layer. As this experiment generates a highly nonequilibrium distribution of phonons, the system temperature cannot be defined, making this comparison only qualitative.

\section{CONCLUSION}

In summary, the study of spatial and temporal coherence of thermally activated phonons unveils a unified description of the wave-particle nature of heat conduction in solids. It reveals the involved transport regimes by revealing characteristic length scales indicating whether phonons behave like diffusing particles or localized/delocalized standing waves. This duality always coexists in any crystal due to the nonlinear character of the interatomic forces. We have proposed a theoretical description of these phenomena and shown how both coherence can be accounted for by extending the definition of the phonon dispersion relations and introducing both a complex frequency and wave vector. Finally, the spatial coherence length provides an additional degree of freedom to control heat properties in condensed matter via the wave nature of phonons, such as energy localization in nanostructured and disordered materials [56].

\section{ACKNOWLEDGMENTS}

We acknowledge the support of the ENIAC program under the NANOTEG Project Grant No. 270789-2 and of the French Ministry of Defense (Direction Générale de l'Armement).
[1] R. Clausius, Philos. Mag. Ser. 4 14, 108 (1857).

[2] J. M. Ziman, Electrons and Phonons: The Theory of Transport Phenomena in Solids, Vol. 20 (Clarendon Press, Oxford, 1960).

[3] R. Peierls, Ann. Phys. 395, 1055 (1929).

[4] A. J. Minnich, M. S. Dresselhaus, Z. F. Ren, and G. Chen, Energy Environ. Sci. 2, 466 (2009).

[5] K. Biswas, J. He, I. D. Blum, C.-I. Wu, T. P. Hogan, D. N. Seidman, V. P. Dravid, and M. G. Kanatzidis, Nature (London) 489, 414 (2012).

[6] R. S. Prasher, X. J. Hu, Y. Chalopin, N. Mingo, K. Lofgreen, S. Volz, F. Cleri, and P. Keblinski, Phys. Rev. Lett. 102, 105901 (2009).

[7] Z. Yan, G. Liu, J. M. Khan, and A. A. Balandin, Nat. Commun. 3, 827 (2012).

[8] Y. S. Ju and K. E. Goodson, Appl. Phys. Lett. 74, 3005 (1999).

[9] A. I. Boukai, Y. Bunimovich, J. Tahir-Kheli, J.-K. Yu, W. A. Goddard III, and J. R. Heath, Nature (London) 451, 168 (2008).

[10] A. I. Hochbaum, R. Chen, R. D. Delgado, W. Liang, E. C. Garnett, M. Najarian, A. Majumdar, and P. Yang, Nature (London) 451, 163 (2008).

[11] B. Poudel, Q. Hao, Y. Ma, Y. Lan, A. Minnich, B. Yu, X. Yan, D. Wang, A. Muto, D. Vashaee, X. Chen, J. Liu, M. S. Dresselhaus, G. Chen, and Z. Ren, Science 320, 634 (2008).

[12] D. G. Cahill, P. V. Braun, G. Chen, D. R. Clarke, S. Fan, K. E. Goodson, P. Keblinski, W. P. King, G. D. Mahan, A. Majumdar, H. J. Maris, S. R. Phillpot, E. Pop, and L. Shi, Appl. Phys. Rev. 1, 011305 (2014).

[13] P. B. Allen and J. L. Feldman, Phys. Rev. B 48, 12581 (1993).

[14] P. B. Allen, J. L. Feldman, J. Fabian, and F. Wooten, Philos. Mag. B 79, 1715 (1999).

[15] Y. M. Beltukov and D. A. Parshin, JETP Lett. 93, 598 (2011).

[16] P. Dean, Proc. Phys. Soc. 84, 727 (1964).

[17] O. N. Dorokhov, Sov. Phys. JETP 58, 606 (1983).

[18] J. L. Feldman, M. D. Kluge, P. B. Allen, and F. Wooten, Phys. Rev. B 48, 12589 (1993).

[19] K. M. Fonseca, Braz. J. Phys. 36, 828 (2006).
[20] C. Gonçalves da Silva and B. Koiller, Solid State Commun. 40, 215 (1981).

[21] J. W. Halley, M. Thorpe, and A. Walker, J. Polym. Sci. 73, 55 (1985).

[22] R. D. Painter and W. M. Hartmann, Phys. Rev. B 13, 479 (1976).

[23] R. Reigada, A. H. Romero, A. Sarmiento, and K. Lindenberg, J. Chem. Phys. 111, 1373 (1999).

[24] M. S. Kushwaha, P. Halevi, L. Dobrzynski, and B. DjafariRouhani, Phys. Rev. Lett. 71, 2022 (1993).

[25] M. Maldovan, Nat. Mater. 14, 667 (2015).

[26] R. Venkatasubramanian, Phys. Rev. B 61, 3091 (2000).

[27] S. Chakraborty, C. A. Kleint, A. Heinrich, C. M. Schneider, J. Schumann, M. Falke, and S. Teichert, Appl. Phys. Lett. 83, 4184 (2003).

[28] V. Rawat, Y. K. Koh, D. G. Cahill, and T. D. Sands, J. Appl. Phys. 105, 024909 (2009).

[29] J. Ravichandran, A. K. Yadav, R. Cheaito, P. B. Rossen, A. Soukiassian, S. J. Suresha, J. C. Duda, B. M. Foley, C.-H. Lee, Y. Zhu, A. W. Lichtenberger, J. E. Moore, D. A. Muller, D. G. Schlom, P. E. Hopkins, A. Majumdar, R. Ramesh, and M. A. Zurbuchen, Nat. Mater. 13, 168 (2014).

[30] M. N. Luckyanova, J. Garg, K. Esfarjani, A. Jandl, M. T. Bulsara, A. J. Schmidt, A. J. Minnich, S. Chen, M. S. Dresselhaus, Z. Ren, E. A. Fitzgerald, and G. Chen, Science 338, 936 (2012).

[31] M. V. Simkin and G. D. Mahan, Phys. Rev. Lett. 84, 927 (2000).

[32] B. C. Daly, H. J. Maris, K. Imamura, and S. Tamura, Phys. Rev. B 66, 024301 (2002).

[33] K. Imamura, Y. Tanaka, N. Nishiguchi, S. Tamura, and H. J. Maris, J. Phys.: Condens. Matter 15, 8679 (2003).

[34] Y. Chen, D. Li, J. R. Lukes, Z. Ni, and M. Chen, Phys. Rev. B 72, 174302 (2005).

[35] K. Termentzidis, P. Chantrenne, J.-Y. Duquesne, and A. Saci, J. Phys.: Condens. Matter 22, 475001 (2010).

[36] J. Garg and G. Chen, Phys. Rev. B 87, 140302(R) (2013).

[37] K.-H. Lin and A. Strachan, Phys. Rev. B 87, 115302 (2013). 
[38] S. C. Huberman, J. M. Larkin, A. J. H. McGaughey, and C. H. Amon, Phys. Rev. B 88, 155311 (2013).

[39] Y. Wang, H. Huang, and X. Ruan, Phys. Rev. B 90, 165406 (2014).

[40] B. Latour, S. Volz, and Y. Chalopin, Phys. Rev. B 90, 014307 (2014).

[41] T. Zhu and E. Ertekin, Phys. Rev. B 90, 195209 (2014).

[42] X. Mu, T. Zhang, D. B. Go, and T. Luo, Carbon 83, 208 (2015).

[43] S. Xiong, B. Latour, Y. Ni, S. Volz, and Y. Chalopin, Phys. Rev. B 91, 224307 (2015).

[44] L. Mandel and E. Wolf, Optical Coherence and Quantum Optics (Cambridge University Press, New York, 1995).

[45] A. Michels, H. Wijker, and H. Wijker, Physica 15, 627 (1949).

[46] A. Bojahr, M. Gohlke, W. Leitenberger, J. Pudell, M. Reinhardt, A. von Reppert, M. Roessle, M. Sander, P. Gaal, and M. Bargheer, Phys. Rev. Lett. 115, 195502 (2015).

[47] A. A. Maradudin and A. E. Fein, Phys. Rev. 128, 2589 (1962).
[48] A. J. C. Ladd, B. Moran, and W. G. Hoover, Phys. Rev. B 34, 5058 (1986).

[49] A. J. H. McGaughey and J. M. Larkin, Annu. Rev. Heat Transfer 17, 49 (2014).

[50] G. Chen, J. Heat Transfer 119, 220 (1997).

[51] See Supplemental Material at http://link.aps.org/supplemental/ 10.1103/PhysRevB.95.214310 to view additional calculations and the movies related to the wave packet dynamics simulations.

[52] P. K. Schelling, S. R. Phillpot, and P. Keblinski, Appl. Phys. Lett. 80, 2484 (2002).

[53] Z. Liang and P. Keblinski, Phys. Rev. B 90, 075411 (2014).

[54] I. Katayama, K. Sato, S. Koga, J. Takeda, S. Hishita, H. Fukidome, M. Suemitsu, and M. Kitajima, Phys. Rev. B 88, 245406 (2013).

[55] L. Lindsay and D. A. Broido, Phys. Rev. B 81, 205441 (2010).

[56] S. Pailhès, H. Euchner, V. M. Giordano, R. Debord, A. Assy, S. Gomès, A. Bosak, D. Machon, S. Paschen, and M. de Boissieu, Phys. Rev. Lett. 113, 025506 (2014). 